\title{
STRD and Near Field Modeling of Metallic Wedges for Optical Detection Systems
}

\author{
Alessandro Massaro and Roberto Cingolani
}

\begin{abstract}
We analyze an accurate approach based on the $\mathrm{Si}$ multaneous Transverse Resonance Diffraction (STRD) modeling. The new method allows to evaluate by means of transmission line circuits the near field generated by a metallic wedge excited by an optical source. The STRD technique is implemented in the rigorous multipole expansion of the Green's function (MEG) theory by providing a modeling of material permittivity detection for wireless micro/nano systems. A good agreement between finite element method (FEM), finite difference time domain (FDTD) and STRD/MEG results is found. Requiring a low computational cost, the proposed modeling is suited for electromagnetic simulators.
\end{abstract}

Index Terms-Metallic wedges modeling, optical detection system, plasmon probes, wireless sensing.

\section{INTRODUCTION}

C ONCENTRATION of optical energy below the diffraction limit has many diverse applications. They include near-field microscopy [1], single-molecule biochemical sensing [2], miniaturized integrated optics [3], high-density data storage [4], non-linear optics [5], surface enhanced Raman scattering or fluorescence [6], and implanted wireless systems [7]. Metallic wedges are suitable for these applications because they can focus the energy in proximity of the discontinuity and they behave as probes for optical detection systems. The optical source is a surface plasmon wave which can be enhanced by a nano/micro grating placed directly on the probe [8]. An example of optical near field detection system is reported in Fig. 1(a) where a transmitter metallic wedge $T_{x}$, excited by an optical wave, radiates the focused electromagnetic field, and, a receiver wedge probe $\mathrm{R}_{x}$ detects the variation of the refractive index $d n$ (dielectric contrast) in a background with refractive index $n$. The distance and the orientation of both probes will be defined in order to distinguish without errors the refractive index variation. Being the near field difficult to evaluate at optical frequencies an accurate approach able to model and design the $T_{x} / R_{x}$ wedge detection system is necessary: the electromagnetic field near the discontinuity of the metallic conductor is irregular and the detection principle becomes impossible. The Simultaneous Transverse Resonance Diffraction

Manuscript received May 06, 2010; revised August 30, 2010; accepted October 20, 2010. Date of publication October 28, 2010; date of current version December 08, 2010.

A. Massaro is with Italian Institute of Technology IIT, Center of Biomolecular Nanotechnologies of Arnesano, Leece 73100, Italy (e-mail: alessandro.massaro@iit.it).

R. Cingolani is with Italian Institute of Technology IIT, Genova 16163, Italy. Color versions of one or more of the figures in this paper are available online at http://ieeexplore.ieee.org.

Digital Object Identifier 10.1109/JLT.2010.2089784

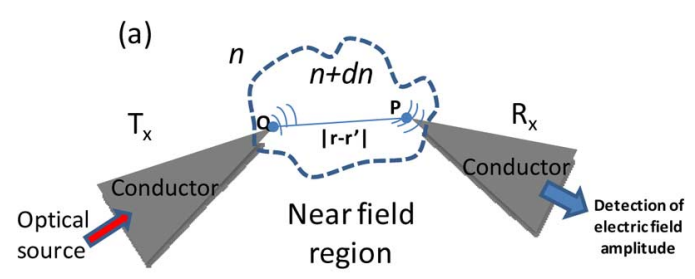

(b)

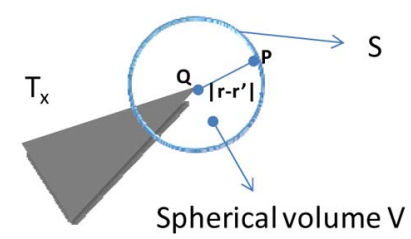

Fig. 1. (a) Schematic diagram of a radiation system with metallic wedges: the transmitter metallic wedge $T_{x}$ is excited by an external optical source and the receiver wedge probe $R_{x}$ detects the variation of the electromagnetic field related to a variation of the refractive index $d n$. (b) Volume $V$ and surface $S$ used for the calculus of the near field.

(STRD) method allows to calculate the near field singularity by distinguishing the near field from the irregular field and consecutively the noise from the signal. The micro-nano scale of antennas requires an accurate modeling especially for near field applications. The traditional numerical approaches such as finite element method (FEM), and finite difference time domain (FDTD) technique require high computational cost for the evaluation of the radiated field in proximity of a dielectric or metallic discontinuity. For this purpose we use the new approach of multipole expansion of the Green's function (MEG) based on the novel principle of STRD [9]: the STRD/MEG was previously applied for the near field evaluation of three dimensional dielectric corner by showing a good convergent solution with a low computational cost and with a reduction of the computational time of $17 / 10$ times if compared with the traditional numerical methods [9]. For the first time in the proposed work we apply the STRD/MEG method to a particular class of antennas obtained as wedge conductors appropriate for plasmon resonance probe systems: the same approach described in [9] for dielectric optical waveguides is implemented and modified in order to consider surface plasmon wave (SPW) excitation propagating along an optical waveguide with thin film metallic layer and radiated by a metallic wedge discontinuity.

At optical frequencies the analysis of the electromagnetic (EM) field radiated from metallic wedges becomes difficult and the traditional FDTD and FEM approaches could fail. Convergent FDTD/FEM solutions, related to the detection of low $d n$ 
values by means of wedge discontinuities, require high computational cost and consecutively complex parallel computing implementations also under the hypothesis of plasmon resonance condition. The new STRD/MEG method overcomes the problem of the high computational cost correlated to the solution accuracy because takes into account the EM singularity, it is easy to implement, and provides good convergent solutions.

We summarize our work in the points listed below. In particular

i) we formulate the STRD modeling of a metallic wedge probe system by implementing the MEG approach;

ii) we calculate the near field for different wedge configurations;

iii) finally we compare the STRD/MEG results with the three dimensional (3-D) FEM and FDTD ones for a radiation system probes operating at $\lambda_{0}=1 \mu \mathrm{m}$.

\section{Multipole ExpAnsion OF THE GREEN's FunCtion}

The radiated electric field at an external point $\mathrm{P}$ of region with refractive index $n$ of Fig. 1 is expressed as [10], [11]

$$
\begin{aligned}
\mathbf{E}(P)= & \frac{1}{j \omega 4 \pi \varepsilon_{c}} \nabla \times \nabla \times\left(\int_{V} \mathbf{J}_{i} G\left(\mathbf{r}, \mathbf{r}^{\prime}\right) d V_{Q}\right. \\
& \left.+\int_{S}(\mathbf{H} \times \hat{n}) G\left(\mathbf{r}, \mathbf{r}^{\prime}\right) d S_{Q}\right) \\
& -\frac{1}{4 \pi} \nabla \times\left(\int_{S}(\hat{n} \times \mathbf{E}) G\left(\mathbf{r}, \mathbf{r}^{\prime}\right) d S_{Q}\right)-\frac{\mathbf{J}_{i}}{j \omega \varepsilon_{c}}
\end{aligned}
$$

where in spherical coordinates the wedge point is at $\mathrm{Q}=\mathrm{Q}\left(\mathrm{r}^{\prime}, \theta^{\prime}, \varphi^{\prime}\right)$, and the field is evaluated at $\mathrm{P}=\mathrm{P}(\mathrm{r}, \theta, \varphi)$ where is located the $\mathrm{R}_{\mathrm{x}}$ probe. The volume $V$ used in (1) is enclosed by the spherical surface $S$ as illustrated in Fig. 1(b). The spherical surface is centred in the singularity point $\mathrm{Q}$.

The permittivity $\varepsilon_{\mathrm{c}}=\mathrm{n}^{2}$ indicates the cladding permittivity. The cladding encloses the wedge. In spherical coordinates, the Green function satisfies the Helmholtz scalar equation and the multipole expansion of the Green's function given by [10]-[22]

$$
G\left(\mathbf{r}, \mathbf{r}^{\prime}\right)=\sum_{l, m} g_{l}\left(r, r^{\prime}\right) Y_{l, m}^{*}\left(\theta^{\prime}, \varphi^{\prime}\right) Y_{l, m}(\theta, \varphi)
$$

We observe that (2) presents a discontinuity peak of the first derivative at the point Q (point of singularity). According with this property the MEG is suited for near field evaluation in proximity of metallic singularities. Moreover the index $l$ indicates the order of the expansion and represents the accuracy of the solution: the convergence of the near field solution can be performed by increasing the order of MEG. A solution of Maxwell's equation in cylindrical coordinates is provided by the following three field components

$$
\begin{aligned}
& E_{\rho}=-j \omega \mu \frac{1}{\rho} \frac{\partial \Phi}{\partial \phi} \\
& E_{\phi}=j \omega \mu \frac{\partial \Phi}{\partial \rho} \\
& H_{z}=k^{2} \Phi
\end{aligned}
$$

where $\Phi$ is a suitable Hertzian potential which satisfies Helmholtz' equation. The field in (3) is TE with respect to the $z$-direction, briefly $\mathrm{TE}^{\mathrm{z}}$, but it is also $\mathrm{TM}$ with respect to (a)

(b)

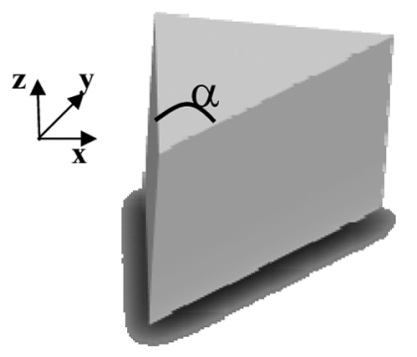

\begin{tabular}{|c|c|c|c|}
\hline Geometry & $v$ & $E_{\rho}, E_{\phi}$ & $H_{z}$ \\
\hline 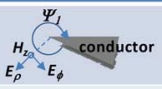 & $v=\pi / \Psi^{\prime}$ & $\propto \rho^{\nu-1}$ & $\propto \rho^{v}$ \\
\hline 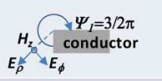 & $v=2 / 3$ & $\propto \rho^{-1 / 3}$ & $\propto \rho^{2 / 3}$ \\
\hline$\underset{E_{\rho}}{\Psi_{2}=2 \pi}{ }_{\text {conductor }}$ & $v=1 / 2$ & $\propto \rho^{-1 / 2}$ & $\propto \rho^{1 / 2}$ \\
\hline
\end{tabular}

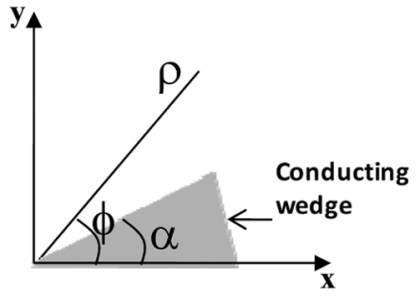

Fig. 2. Perfectly conducting wedge infinite in the $z$-direction. (a) 3-D-view (b) geometry in the transverse plane also showing the cylindrical coordinate system.

TABLE I

BeHAVIOUR OF TE $^{z}$ FIELD NEAR A METALLIC WEDGE

the $\phi$-direction (thus $\mathrm{TM}^{\phi}$ ). A similar modal analysis can be carried out for $\mathrm{TM}^{\mathrm{z}}$ (i.e., $\mathrm{TE}^{\phi}$ ) fields characterized by the following components

$$
\begin{aligned}
& H_{\rho}=j \omega \varepsilon \frac{1}{\rho} \frac{\partial \Phi}{\partial \phi} \\
& H_{\phi}=-j \omega \varepsilon \frac{\partial \Phi}{\partial \rho} \\
& E_{z}=k^{2} \Phi .
\end{aligned}
$$

Equations (3) and (4) are used in order to calculate the total electric field (1) near the metallic wedge by using the relationships which convert the cylindrical coordinates to spherical coordinates. In particular, (3) and (4) are the fields used in (1) as $\mathbf{E}$ and $\mathbf{H}$, they takes into account the singularity of the EM field and allow to distinguish the TE analysis from the TM one. The TE and the TM approaches are related to the TE or TM polarization of the source.

\section{Simultaneous Transverse Resonance DifFraction MODELING AND SINGULARITY EVALUATION}

It is well recognized that, in many cases, boundary and radiation conditions alone are not sufficient to determine the solution uniquely [9], [23], [24]. In any case it is possible to construct several different fields which satisfy these conditions [9]. In particular, by considering the metallic wedge reported in Fig. 2, the MEG approach well matchs the radiation conditions especially for the near field calculation, but an accurate evaluation of the potential $\Phi$ is requested.

The $\rho$-dependence of the $\Phi$ potential can be written, in general, as combination of Bessel and Hankel functions. In particular, since no source is present in $\rho=0$ the excitation arrives in $\mathrm{Q}$ but it is not localized in Q), the $\rho$-dependence is completely 
TABLE II

Behaviour of $\mathrm{TM}^{z}$ Field NeAr a Metallic Wedge

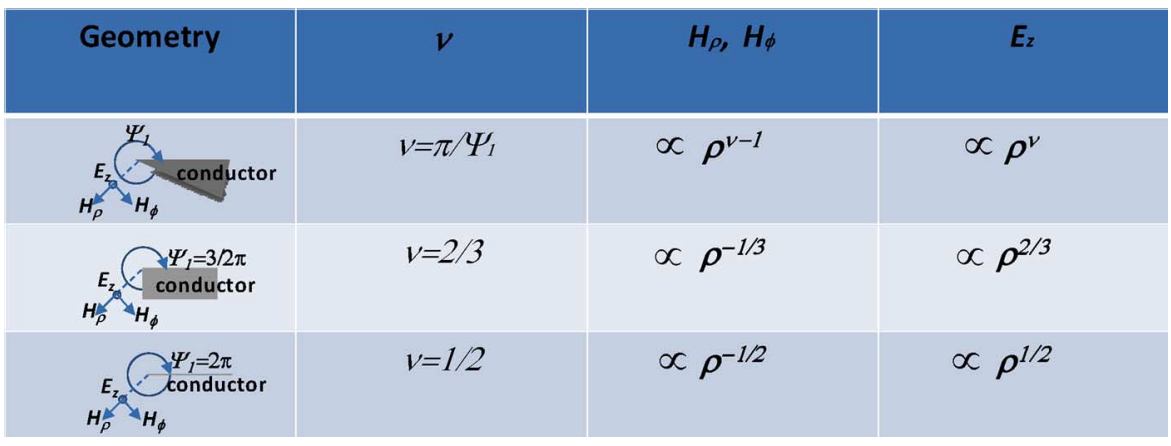

(a)

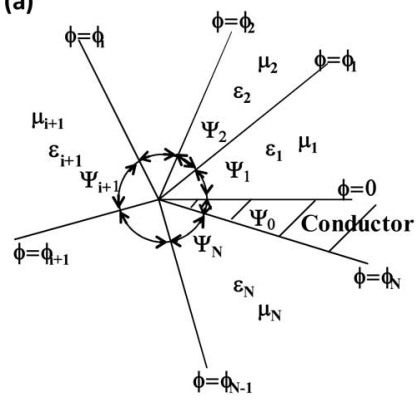

(c)

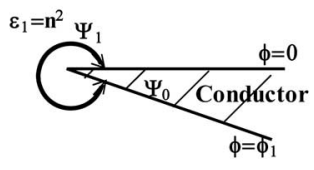

(b)

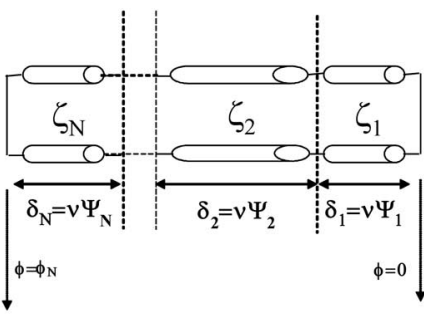

(d)

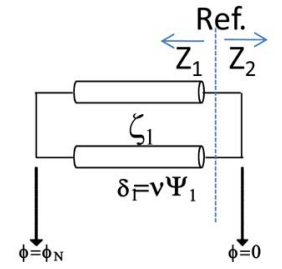

Fig. 3. (a) A perfect conducting wedge and $\mathrm{N}$ dielectric wedges. The $z$ axis is along the common wedge and normal to the figure. (b) Equivalent transmission line circuit: $\delta_{\mathrm{i}}$ represent the electric lengths and $\zeta_{\mathrm{i}}$ the characteristic impedance, respectively. (c) conducting wedge embedded in a material with refractive index $n$, and (d) equivalent transmission line circuit.

described by Bessel functions. For this reason, near the edge, we may use the small argument approximation of the Bessel function by the trigonometric dependence on the angle $\phi$ thus providing the following form of the potential

$$
\Phi(\rho, \phi)=\frac{1}{k_{0}^{2}} \rho^{\nu}\left[A_{i} \sin (\nu \phi)-B_{i} \cos (\nu \phi)\right]
$$

where $k_{0}=2 \pi / \lambda_{0}$ is the free space wave number and $\lambda_{0}$ is the optical working wavelength, the near field will be characterized by the singularity $\nu$ which can be calculated by a circuital approach. If the metallic wedge is embedded in $\mathrm{N}$ dielectric materials, as indicated in Fig. 3(a), the circuital approach takes into account the electromagnetic field propagating along the cascade of transmission lines of Fig. 3(b). Each transmission line is characterized by an electric length $\delta_{\mathrm{i}}=\nu \Psi_{\mathrm{i}}$ (which is function of the angle $\Psi_{\mathrm{i}}$ related to the dielectric material $\varepsilon_{\mathrm{i}}$ ), and by a characteristic impedance $\zeta_{\mathrm{i}}$ [12]. The simultaneous resonance of the transmission lines will provide the singularity $\nu$ of the electromagnetic field. The resonance condition is performed by annulling the total impedance of the circuit with respect to a reference plane [12]. If the conductor is embedded in a single dielectric material $\varepsilon_{1}=\varepsilon_{\mathrm{c}}=\mathrm{n}^{2}$ as reported in Fig. 3(c), the equivalent transmission line circuit of the metallic edge will be a single transmission line enclosed in two short circuit (see Fig. 3(d)).

Let us consider for simplicity a transverse electric TE-polarized $\left(H_{z}, E_{\rho}, E_{\varphi}\right)$ field. By defining the following equivalent voltages and currents related to the scheme of Fig. 3(a)

$$
\begin{aligned}
V_{i} & =\frac{1}{\varepsilon_{i}}\left[A_{i} \cos \left(\nu \phi_{i}\right)+B_{i} \sin \left(\nu \phi_{i}\right)\right] \\
I_{i} & =\left[A_{i} \sin \left(\nu \phi_{i}\right)-B_{i} \cos \left(\nu \phi_{i}\right)\right] \quad \mathrm{i}=1, \ldots, \mathrm{N}
\end{aligned}
$$

and by making use of the continuity of $H_{\mathrm{Z}}$ and $E_{\rho}$ components we derive the following transmission matrix $(A B C D)$ representation [25]

$$
\left(\begin{array}{c}
V_{i+1} \\
I_{i+1}
\end{array}\right)=\left(\begin{array}{cc}
\cos \left(\nu \phi_{i}\right) & \zeta_{i} \sin \left(\nu \phi_{i}\right) \\
\zeta_{i}^{-1} \sin \left(\nu \phi_{i}\right) & \cos \left(\nu \phi_{i}\right)
\end{array}\right)\left(\begin{array}{c}
V_{i} \\
I_{i}
\end{array}\right)
$$

where $\zeta_{\mathrm{i}}=1 / \varepsilon$ is the normalized $\mathrm{TM}^{\phi}$ characteristic impedance. By introducing the load impedance $\mathrm{Z}_{\mathrm{i}}=\mathrm{V}_{\mathrm{i}} / \mathrm{I}_{\mathrm{i}}$ and $T_{i}=\tan \left(\nu \Psi_{i}\right)$ and by using the standard formula for the impedance transformation along a transmission line:

$$
Z_{i}=\zeta_{i} \frac{Z_{i-1}+j \zeta_{i} T_{i}}{\zeta_{i}+j Z_{i-1} T_{i}} \quad i=1,2, \ldots, \mathrm{N}
$$

we can calculate the simultaneous resonance condition of the total impedance $Z_{1}+Z_{2}=$ in the case of $N=1$ (case of Fig. 3(c)) where $Z_{1}$ and $Z_{2}$ are the impedances calculated starting from the reference plane of Fig. 3(d). In this case we have that $\mathrm{T}_{1}=0$, that is

$$
\nu=\frac{\pi}{\Psi_{1}}
$$

By applying the resonance condition to different wedge geometries we obtain the singularity $\nu$ of the $\mathrm{TE}^{\mathrm{Z}}$ and $\mathrm{TM}^{\mathrm{Z}}$ near field reported in Tables I and II, respectively. We note that according with Fig. 2 the angle $\Psi_{1}$ is equal to $2 \pi-\alpha$.

\section{Source Characterization: Surface Plasmon WaVe AND PLASMON RESONANCE}

According to (2) the STRD-MEG is based on the expansion of spherical harmonics which allows to obtain a very accurate solution regarding radiation problems. These functions must be matched with the source in order to improve the exact solution of the physical problem. In particular for the STRD-MEG approach we use as input field of the antenna a surface plasmon 

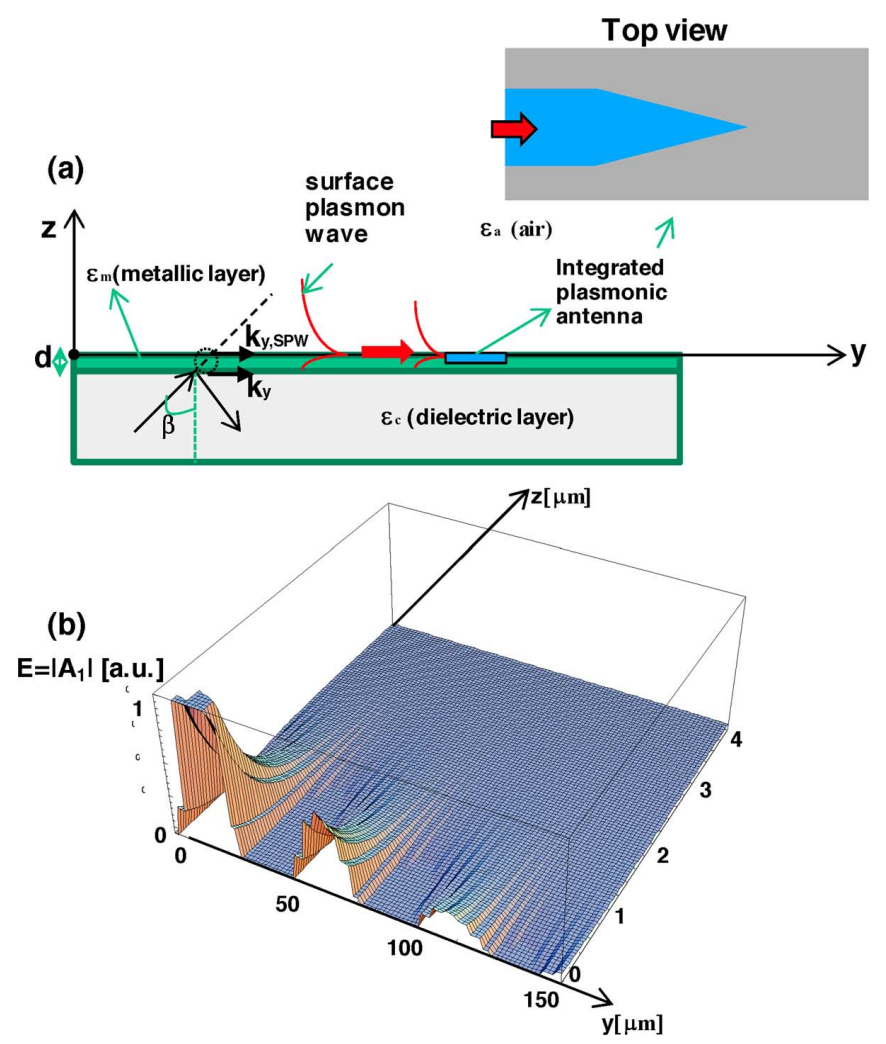

Fig. 4. (a) Plasmon waveguide and excitation of a surface plasmon wave impingings on an integrated plasmonic antenna (metallic wedge). (b) Example of SPW for a waveguide with $\varepsilon_{\mathrm{c}}=3.1, \varepsilon_{\mathrm{a}}($ air $)=$ $1, \mathrm{~d}$ (thickness of a gold layer) $=50 \mathrm{~nm}$, and operating at $\lambda_{0}=1 \mu \mathrm{m}$.

wave (SPW) generated by the plasmon waveguide of Fig. 4(a). Assuming as source an electric field, impinging with an angle $\beta$ on the thin film metallic layer reported in Fig. 4(a), the resulting surface electromagnetic wave will have the following general form

$$
\begin{aligned}
& \mathbf{A}_{1}=\mathbf{A}_{10} e^{i\left(\mathbf{k}_{z 1} z+\mathbf{k}_{y, \mathrm{EW}} y-\omega t\right)} \quad \mathrm{z}>0 \\
& \left.\mathbf{A}_{2}=\mathbf{A}_{20} e^{i\left(\mathbf{k}_{z 2} z+\mathbf{k}_{y}, \mathrm{SPW} y-\omega t\right)}\right] \quad \mathrm{z}<0
\end{aligned}
$$

where $\mathbf{A}$ stands for $\mathbf{E}$ and $\mathbf{H}, k_{z 1}$ and $k_{z 2}$ are the wavevectors in the $z$-direction, $k_{y}$,EW and $k_{y}$,SPW are those in they $x$-direction and $\omega$ is the angular frequency. The incident light coming from the dielectric material reflects at the metallic interface and localized light, called evanescent field (EW), occurs. The EW excites the surface plasmon wave reported in Fig. 4(b) (the plot is obtained by fixing the geometrical and the optical parametrs) which is propagating along the surface of the metal film and thus, a part of the incident light is absorbed. The light intensity of the reflected ray will depend on the incidence angle $\theta$. The propagation constant of the EW is expressed as

$$
k_{y, \mathrm{EW}}=\frac{2 \pi}{\lambda_{0}} \sqrt{\varepsilon_{c}} \sin \theta
$$

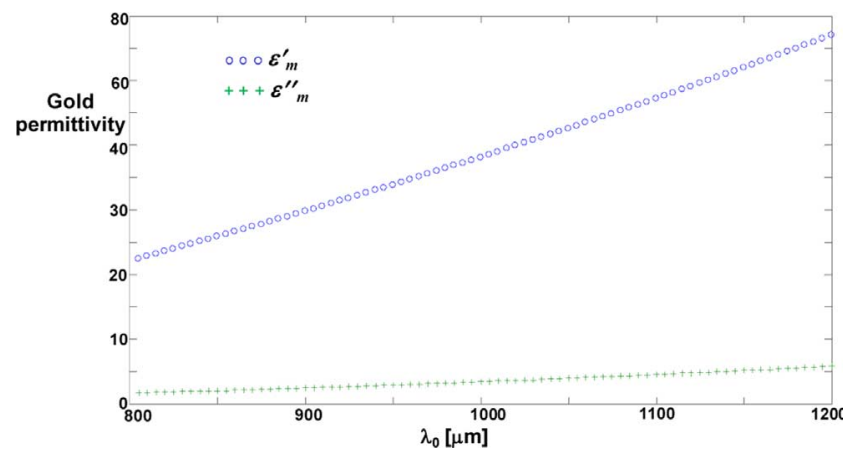

Fig. 5. Calculated gold permittivity versus the working wavelength $\lambda_{0}$.
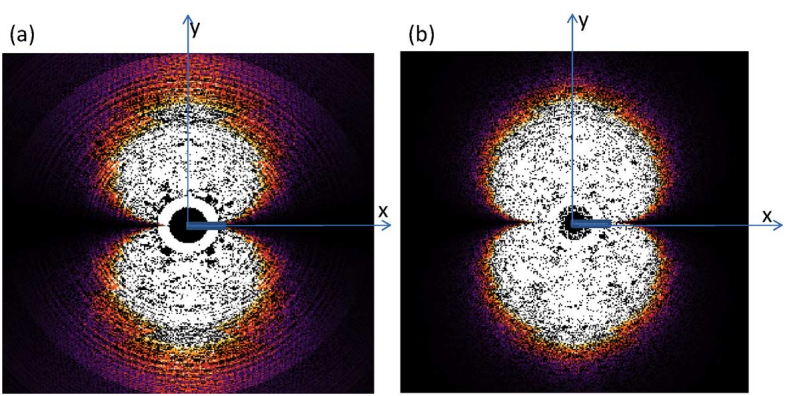

Fig. 6. $\mathrm{TE}^{\mathrm{z}}$ near field radiation pattern of the near electric field for a conductor wedge with $\Psi_{1}=2 \pi$ and for (a) $l=1$ and (b) $l=3$. The plot are calculated by fixing $z=0.1 \mathrm{~nm}$.

where $\lambda_{0}$ is the working wavelength. The propagation constant of the SPW wave is given by the following expression [26], [27]:

$$
\begin{aligned}
k_{d}= & \frac{2 \pi}{\lambda_{0}}\left(\frac{\varepsilon_{a} \varepsilon_{m}}{\varepsilon_{a}+\varepsilon_{m}}\right)^{1 / 2} \\
k_{r}= & -k_{d}\left(\frac{2}{\varepsilon_{a}-\varepsilon_{m}}\right)\left(\frac{\varepsilon_{a} \varepsilon_{m}}{\varepsilon_{a}+\varepsilon_{m}}\right)^{3 / 2} \\
& \times \exp \left\{i \frac{4 \pi d}{\lambda_{0}} \frac{\varepsilon_{m}}{\left(\varepsilon_{a}+\varepsilon_{m}\right)^{1 / 2}}\right\}
\end{aligned}
$$

where $k_{d}$ is the dispersion relation of the surface plasmon wave of a metal-vacuum interface in a one-sharp-boundary model, $k_{r}$ is the perturbation to $k_{d}$ due to the physical volume of the metal defined by the thickness $d$, and the complex dielectric function of a metallic film can be expressed in the following form:

$$
\varepsilon_{m}(\omega)=\varepsilon_{m}^{\prime}(\omega)+i \varepsilon_{m}^{\prime \prime}(\omega)
$$

When (11) and (12) satisfy the following equation, the surface plasmon resonance occurs and a part of incident light is absorbed

$$
k_{y, \mathrm{SPW}}=k_{d}+k_{r}
$$

At the resonance condition the infinitely metal conductor placed at the end of the waveguide will be coupled and will be radiated as explained in the previous Section III. 


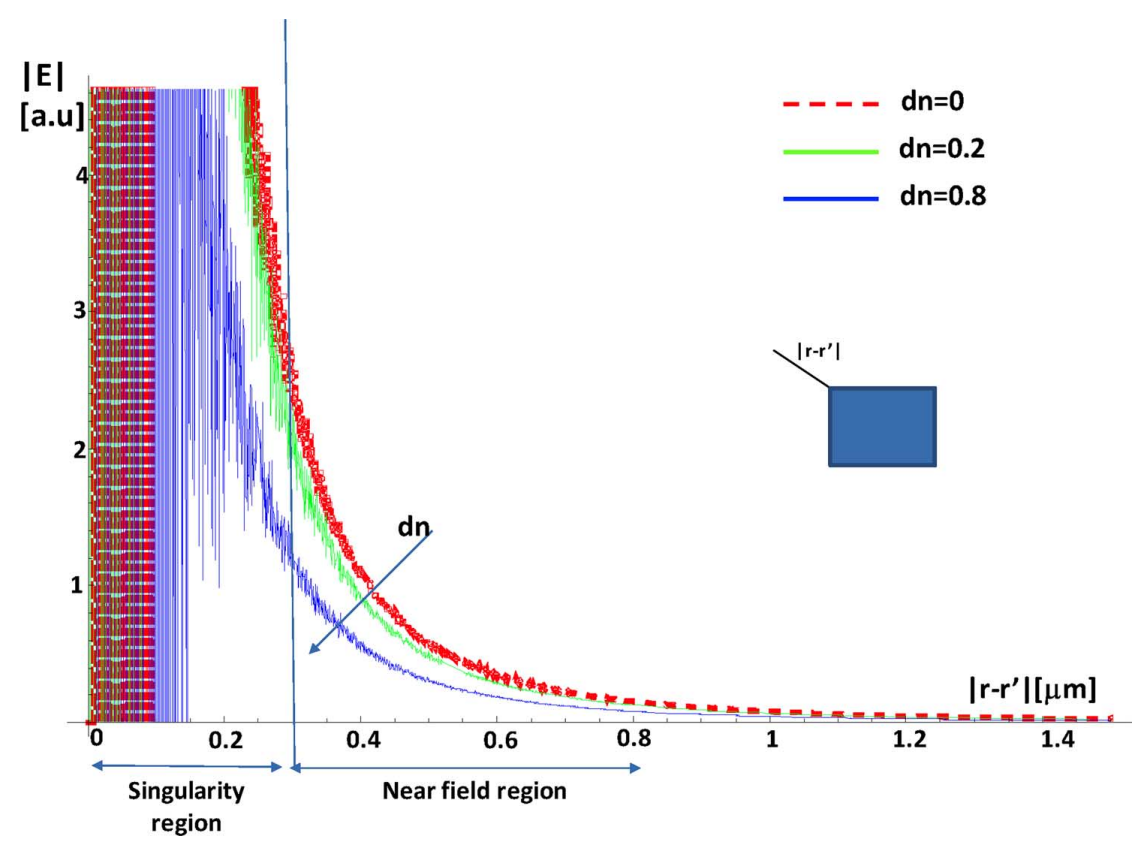

Fig. 7. $90^{\circ}$ metallic wedge: total $\mathrm{TE}^{\mathrm{z}}$ electric field versus $\left|\mathrm{r}-\mathrm{r}^{\prime}\right|$ and versus different variations $d n$ of refractive index. The order of the MEG expansion is $l=3$. Inset: schematic plot of the distance $\left|\mathrm{r}-\mathrm{r}^{\prime}\right|$.

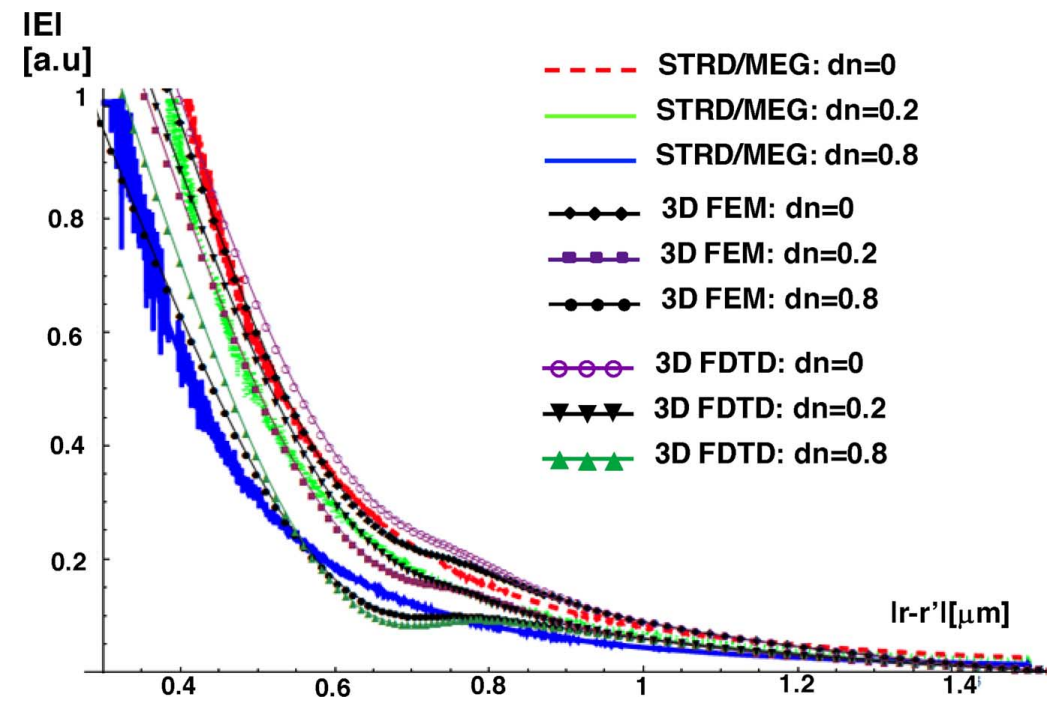

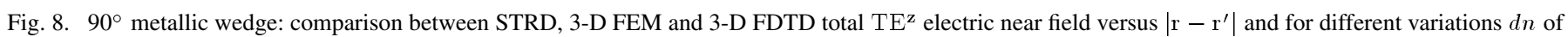
refractive index. The order of the MEG expansion is $l=3$.

\section{RESULTS AND DisCUSSIONS}

As application of the STRD approach implemented in MEG theory (STRD/MEG) we fix the working wavelength to $\lambda_{0}=$ $1 \mu \mathrm{m}$ (InGaAsP laser source) and we implement (1) by means of a FORTRAN code. We consider a gold plasmonic antenna. The permittivity is calculated by means of the Brendel-Bormann model [26] (see Fig. 5) by obtaining at $\lambda_{0}=1 \mu \mathrm{m} \varepsilon_{\mathrm{m}}^{\prime}=38.15$ and $\varepsilon_{\mathrm{m}}^{\prime \prime}=3.474$. In order to observe the accuracy of the STRD near field solution with respect to the order $l$ of the MEG expansion of (2), we calculate in Fig. 6 the radiation pattern in the $x y$-plane of the $\mathrm{TE}^{\mathrm{z}}$ field for a conductor with $\alpha=0$ and for different $l$ values. We note that the infinitely thin metal conductor $(\alpha=0)$ represents a critical case regarding the convergence of the near field solution, therefore it is useful in order to fix the minimum value of the order $l$ which will perform a close solution for all the geometrical configurations with $\alpha \neq 0$. The TE ${ }^{\mathrm{Z}}$ assumption is due to the hypothesis of $\mathrm{TE}^{\mathrm{z}}$ optical source excitation, and the total electric field is calculated by (1), by Table I, by supposing the impressed currents $\mathbf{J}_{\mathrm{i}}=0$, and by integrating over the total surface $\mathrm{S}$ and over the total volume $V$ reported in Fig. 1(b). Equations (3), calculated by (5) and (9), are implemented as the $\mathbf{E}$ and $\mathbf{H}$ fields indicated in (1).

We observe from Fig. 6(a) that the solution with $l=1$ is weakly accurate, while the solution with $l=3$ reported in Fig. 6(b) is convergent. The uniform intensity of the electric field distribution illustrated in the $x y$ plane of Fig. $6(\mathrm{~b})$ defines a clear shape (similar to an eight) of the near field radiation pattern. This does not happen in the case of Fig. 6(a) where the shape of the field intensity is irregular: in this case discontinuous 
regions of intensities are observed as fringes of the boundary, this allows to not establish during the design process the correct position of the point $\mathrm{P}$ in the $x y$ plane (point where can be located the receiver wedge probe $\mathrm{R}_{\mathrm{x}}$ ).

For this purpose the choice of the order for the conductor edges must be at least $l=3$. We consider now the transmitter/receiver system of Fig. 1 and the case of $90^{\circ}$-metal edge $(\alpha=$ $90^{\circ}$ ) relevant for many practical applications, and, we suppose that the receiver probe $R_{x}$ is placed at a distance $\left|r-r^{\prime}\right|$ which depends on the working wavelength and on the near field region. In order to analyze the probe sensitivity we consider the solution (1) for materials characterized by different dielectric contrasts $d n$. Figs. 7 and 8 prove that the STRD/MEG approach allows to distinguish the near field amplitudes also for low dielectric contrasts. Observing Fig. 7 we conclude that in this case the receiver probe $\mathrm{R}_{\mathrm{x}}$ must be located at a distance between 0.3 and $0.8 \mu \mathrm{m}$ (near field region where it is possible to distinguish the variation of the electric field amplitude). The oscillations observed in Fig. 7 below 0.3 are related to the singularity region characterized by discontinuities of the EM field near the singularity point Q. In the singularity no very closed solutions can be performed. Smaller oscillations can be obtained by increasing the order $l$ of the expansion.

Fig. 8 validates the convergence of the STRD/MEG approach by means of the comparison with 3-D FEM results: a good agreement between 3-D FEM and STRD/MEG results is found. We use for the comparison a properly designed 3-D FEM tool typically used for the design of micrometric structures [9]. The FEM near field region is the region closest to the source, and the 3-D FEM electric field $\mathbf{E}(\mathrm{x}, \mathrm{y}, \mathrm{z})$ external to the region bounded by a closed spherical surface may be written as [24]

$$
\begin{aligned}
\mathbf{E}(x, y, z)=\int_{S}\left(\left(j \omega \mu_{0} \mathbf{H}_{\tan }\right) G\right. \\
\left.\quad+\left(\mathbf{E}_{\tan } \times \nabla G\right)+\left(\mathbf{E}_{\text {normal }} \nabla G\right)\right) d S
\end{aligned}
$$

where $S$ represents the radiation boundary surface, $j$ is the imaginary unit, $\mathbf{H}_{\tan }$ is the component of the magnetic field that is tangential to the surface, $\mathbf{E}_{\text {normal }}$ is the component of the electric field that is normal to the surface, $G$ is the free space Green's function, given by

$$
G=\frac{e^{-j k_{0}\left|\mathbf{r}-\mathbf{r}^{\prime}\right|}}{\left|\mathbf{r}-\mathbf{r}^{\prime}\right|}
$$

being $k_{0}$ the free space wave number, and, $\mathbf{r}$ and $\mathbf{r}^{\prime}$ representing field points and source points on the surface, respectively. The 3-D FEM tool simulates, as in the STRD/MEG case, the radiation of the $90^{\circ}$-metal edge of a perfect metallic square plate with side of $1 \mu \mathrm{m}$ by using a TE ${ }^{\mathrm{z}}$ source. The 3-D FEM modeling of the source is illustrated in Fig. 9(a). According with Fig. 7, a clear variation of $d n$ (corresponding to a near field variation) is checked around $\left|\mathbf{r}-\mathbf{r}^{\prime}\right|=0.4 \mu \mathrm{m}$. For this reason the FEM $R_{\mathrm{x}}$ probe is placed at a distance of $0.4 \mu \mathrm{m}$ along the $y$ direction of Fig. 9(a).

In Fig. 9(b) we show the 3-D FEM total electric field excited by $T_{x}$ and coupled with $R_{x}$. The total 3-D FEM electric field follows the STRD/MEG radiation pattern illustrated in the inset of Fig. 9(b). (a)

(b)

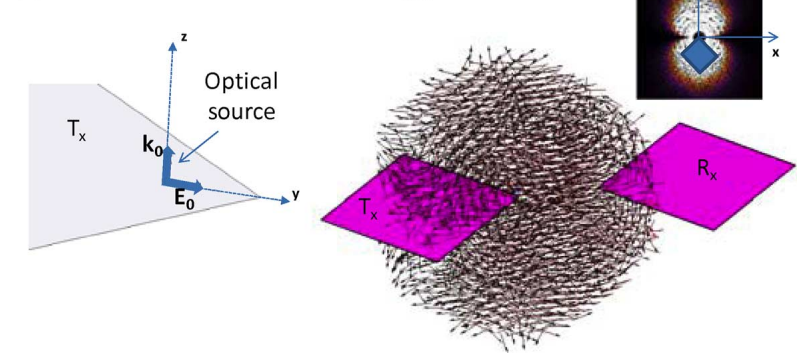

Fig. 9. 3-D FEM modeling of $90^{\circ}$ metallic wedge. (a) $\mathrm{TE}^{\mathrm{z}}$ optical source source. (b) $\mathbf{E}$ total vector field coupled with the $\mathrm{R}_{\mathrm{x}}$ probe; inset: STRD/MEG radiation pattern with $l=3$.

TABLE III

COMPARISON AMONG STRD/MEG, 3-D FEM AND 3-D FDTD COMPUTATIONAL Time. THE SyMBOL “*” MEANS: PARALlel COMPUTING IS

\begin{tabular}{|c|c|c|c|}
\hline $\begin{array}{c}d n \\
\text { Sensitivity }\end{array}$ & STRD/MEG & 3D FEM & 3D FDTD \\
\hline $1 \div 10$ & $\begin{aligned} \cong & 3 \mathrm{~min} . \\
& (l=1)\end{aligned}$ & $\cong 20 \mathrm{~min}$. & $\cong 35 \mathrm{~min}$. \\
\hline $10^{-1}$ & $\begin{aligned} \cong & 50 \mathrm{~min} . \\
& (l=3)\end{aligned}$ & $\cong 10 \mathrm{~h}$ & $\cong 12 \mathrm{~h}$. \\
\hline $10^{-2}$ & $\begin{array}{c}\cong 1 \text { h. } 12 \mathrm{~min} . \\
(l=4)\end{array}$ & $\cong 13 \mathrm{~h}$. & $\cong 16 \mathrm{~h}$. \\
\hline $10^{-3}$ & $\begin{array}{c}\cong 1 \mathrm{~h} .35 \mathrm{~min} . \\
(l=5)\end{array}$ & $*$ & * \\
\hline $10^{-4}$ & $\begin{array}{c}\cong 1 \text { h. } 55 \text { min. } \\
(l=6)\end{array}$ & $*$ & $*$ \\
\hline $10^{-5}$ & $\begin{array}{c}\cong 2 \mathrm{~h} .20 \mathrm{~min} . \\
(l=7)\end{array}$ & $*$ & $*$ \\
\hline $10^{-6}$ & $\begin{array}{c}\cong 2 \mathrm{~h} .40 \mathrm{~min} . \\
(l=8)\end{array}$ & $*$ & * \\
\hline $10^{-7}$ & $\begin{array}{c}\cong 2 \text { h. } 58 \text { min. } \\
(l=9)\end{array}$ & $*$ & $*$ \\
\hline
\end{tabular}
REQUiRED. THE DifFERENCE BETWEEN THE CASE OF $l=1$ AND $l=3$ Is DUE TO THE IMPLEMENTATION OF THE CUMULATIVE SUM FunCtion

The efficiency of the STRD model is shown in Table III, where we compare the computational (CPU)-time for STRD/MEG, 3-D FEM and 3-D FDTD methods (with a 1-GHz PC, 512/M-RAM). The tetrahedral elements used in the 3-D FEM simulation and the FDTD rectangular mesh have the same length of the space-step of STRD/MEG. The multipole expansion order $l=3$ employed for the plots of Fig. 6 provides a good convergent solution with a low computational cost, by decreasing the computational time of about 10 times. The order $l=1$ requires only 3 minutes but the solution is inaccurate. As indicated in the same table, by increasing the order $l$ it is possible to evaluate the effect of very low variations of $d n$ : the numerical detection sensitivity represents the accuracy of the STRD/MEG solution and can be performed in short time (about 3 hours are necessary in order to obtain a sensitivity of $10^{-7}$ ) without parallel computing implementations. For an high sensitivity of FEM and FDTD approaches, the parallel calculus is required: FEM and FDTD do not implement the irregular field due to singularity $\nu$ and expanded by the multipole expansion of the Green's function, and, consecutively, very fine meshes are required by overcoming the PC limits. We observe that the 
STRD/MEG computational cost can be further decreased by using an implementation suitable with the parallel calculus.

\section{CONCLUSION}

We presented in this work a new theoretical model STRD/MGE which provides with a good accuracy and low computational cost the near field generated by a generic metallic edge. The proposed approach is applied for the first time to a well defined metallic probe system by showing an high sensitivity also for the detection of low dielectric contrasts. The STRD/MEG is suited for standard simulators and is able to design plasmon probe for wireless systems.

\section{REFERENCES}

[1] K. Hoshino, A. Gopal, and X. Zhang, "Near-field scanning nanophotonic microscopy-Breaking the diffraction limit using integrated nano light-emitting probe tip," IEEE J. Sel. Topics Quantum Electron., vol. 5, no. 5, pp. 1393-1399, Oct. 2009.

[2] J. Wenger and H. Rigneault, "Photonic methods to enhance fluorescence correlation spectroscopy and single molecule fluorescence detection," Int. J. Mol. Sci., vol. 11, pp. 206-221, 2010.

[3] N. A. Issa and R. Guckenberger, "Optical nanofocusing on tapered metallic waveguides," Plasmonics, vol. 2, pp. 31-37, 2007.

[4] G. A. Shaw, J. S. Trethewey, A. D. Johnson, W. J. Drugan, and W. C. Crone, "Thermomechanical high-density data storage in a metallic material via the shape-memory effect," Adv. Mater., vol. 17, pp. 1123-1127, 2005.

[5] T. Ichimura, N. Hayazawa, M. Hashimoto, Y. Inouye, and S. Kawata, "Application of tip-enhanced microscopy for nonlinear Raman spectroscopy," Appl. Phys. Lett., vol. 84, no. 10, Mar. 2004.

[6] Y. S. Huh, A. J. Chung, and D. Erickson, "Surface enhanced Raman spectroscopy and its application to molecular and cellular analysis," Microfl. Nanofl., vol. 6, pp. 285-297, 2009.

[7] R. N. Simons, F. A. Miranda, and J. D. Wilson, "Wearable wireless telemetry system for implantable Bio-MEMS sensor," in Proc. 28th IEEE EMBS Conf., 2006, pp. 6245-6248.

[8] C. Ropers, C. C. Neacsu, T. Elsaesser, M. Albrecht, M. B. Raschke, and C. Lienau, "Grating-coupling of surface plasmons onto metallic tips: A nanoconfined light source," Nanolett., vol. 7, no. 9, pp. 2784-2788, 2007.

[9] A. Massaro, L. Pierantoni, R. Cingolani, and T. Rozzi, "A new analytical model of diffraction by 3-D-dielectric corners," IEEE Trans. Antennas Propagat., vol. 57, no. 8, pp. 2323-2330, Aug. 2009.

[10] C. G. Someda, Onde Elettromagnetiche. Torino, Italy: UTET Torino, 1996.

[11] V. I. Okhmatovski and A. C. Cangellaris, "Efficient calculation of the electromagnetic dyadic Green's function in spherical layered media," IEEE Trans. Antennas Propagat., vol. 51, no. 12, pp. 3209-3220, Dec. 2003.

[12] T. Rozzi and M. Mongiardo, Open Electromagnetic Waveguides. London, U.K.: IEE, 1997, pp. 21-50.

[13] L. Pierantoni, A. Massaro, and T. Rozzi, "Accurate modeling of TE/TM propagation and losses of integrated optical polarizer," IEEE Trans. Microw. Theory Tech., vol. 53, no. 6, pp. 1856-1862, Jun. 2005.

[14] I. V. Lindell, "Complex space multipole expansion theory with application to scattering from dielectric bodies," IEEE Trans. Antennas Propagat., vol. AP-35, no. 6, pp. 683-689, Jun. 1987.
[15] W. W. Lui, C.-L. Xu, W.-P. Huang, K. Yokoyama, and S. Seki, "Fullvectorial mode analysis with considerations of field singularities at corners of optical waveguides," J. Lightw. Technol., vol. 17, no. 8, pp. 1509-1513, Aug. 1999.

[16] A. Hausner, "Multipole expansion of light vector," IEEE Trans. Visual. Comp. Graph., vol. 3, no. 1, pp. 12-22, Jan.-Mar. 1997.

[17] J. Song and W. C. Chew, "Error analysis for the truncation of multipole expansion of vector Green's functions," IEEE Microw. Wireless Compon. Lett., vol. 11, no. 7, pp. 311-313, Jul. 2001.

[18] Y. Chen, T. L. Simpson, and T. Q. Ho, "Higly efficient technique for solving radiation and scattering problems," Proc. Inst. Elect. Eng. H, vol. 139, no. 1, pp. 7-10, Feb. 1992.

[19] J. S. Gardner, "Uniform asymptotic expansion of the associated Legendre function to leading term for complex degree and integral order," IEEE Trans. Antennas Propag., vol. 55, no. 6, pp. 1797-1803, Jun. 2007.

[20] R. Cicchetti and A. Faraone, "Analysis of open-ended circular waveguide using physical optics and incomplete Hankel functions formulation," IEEE Trans. Antennas Propag., vol. 55, no. 6, pp. 1887-1892, Jun. 2007.

[21] P. M. Morse and H. Feshbach, Methods of Theoretical Physics, 5th ed. New York: McGraw-Hill, 1953, pp. 1273-1283.

[22] G. R. Hadley, "High-accuracy finite_difference equations for dielectric waveguide analysis II: Dielectric corner," J. Lightw. Technol., vol. 20, no. 7, pp. 1221-1231, Jul. 2002.

[23] J. Van Bladel, Electromagnetic Fields. New York: McGraw-Hill, 1964.

[24] S. Caorsi, D. Moreno, and F. Sidoti, "Theoretical and numerical treatment of surface integrals involving the free-space Green's function," IEEE Trans. Antennas Propagat., vol. 41, no. 9, pp. 1296-1301, 1993.

[25] D. M. Pozar, Microwave Engineering, 2nd ed. New York: Wiley, 1998.

[26] A. Rakic, A. B. Djurisic, J. M. Elazar, and M. L. Majewski, "Optical properties of metallic films for vertical-cavity optoelectronic devices," Appl. Opt., vol. 37, no. 22, pp. 5271-5283, 1998.

[27] K. Kurihara and K. Suzuki, "Theoretical understanding of an absorption-based surface plasmon resonance sensor based on Kretchmann's theory," Anl. Chem., vol. 74, no. 3, pp. 696-701, 2002.

Alessandro Massaro received the Laurea degree in electronic engineering and the Ph.D. degree in telecommunication engineering from the Università Politecnica delle Marche, Ancona, Italy, in 2001 and 2004, respectively.

From 2004 to 2006 he worked as Research Scientist (postdoc) in the Department of Electromagnetism and Bioengineering at Università Politecnica delle Marche. In 2006, he spent two years in Research and Development at medical and industrial optics industry (endoscope design and optical systems). He worked for two years with National Nanotechnology Laboratory of CNR-INFM as principal investigator. He is currently team leader in Robotics Lab. 1 of the Center of Bio-Molecular Nanotechnology of Italian Institute Technology (IIT), Arnesano, Lecce, Italy. His research interests are in the design and modeling of photonic bandgap circuits, in the development of computer aided design (CAD) tools in the area of integrated optics, MEMS/NEMS technology and systems, and smart material modeling.

Dr. Massaro is a member of the European Microwave Association (EuMA).

Roberto Cingolani was born in Milan, Italy, in 1961. He received the Ph.D. degree in physics at Scuola Normale Superiore di Pisa, Italy,

He was then a Staff Member of Max Planck Institute FKF-Stuttgart, Germany, from 1989-1991. He was visiting scientists at Tokyo University, Tokyo, Japan, in 1997, and appointed Full Professor of experimental physics at University of Salento in 2000. He is Scientific Director of the Italian Institute of Technology since 2004. He is author and coauthor of more than 500 papers in international journals and he holds 20 patents. His research activity includes nanotechnology and nanofabrication, semiconductor physics and devices, molecular organic nanostructures and devices, and bio-nanotechnologies. 